\title{
Induction of Enzyme Activities in Tuberose Plants Treated with Antagonists and Organic Fungicide under Artificial Inoculation of Sclerotium rolfsii Sacc.
}

\author{
G. Ragavi ${ }^{1}$, M.L. Mini ${ }^{2 *}$, I. Yesuraja ${ }^{3}$ and K. Sethuraman ${ }^{4}$ \\ ${ }^{1}$ Department of Plant Pathology, ${ }^{2}$ Department of Biotechnology, Agricultural College and \\ Research Institute, Tamil Nadu Agricultural University, Madurai-625104, Tamil Nadu, India \\ ${ }^{3}$ Horticulture Research Station, Thadiyankudisai, Dindigul - 624 212, Tamil Nadu, India \\ ${ }^{4}$ Maize Research Station, Vagarai, Dindigul - 624 613, Tamil Nadu, India \\ *Corresponding author
}

\begin{tabular}{|c|c|}
\hline & A B S T R A C T \\
\hline & \multirow{8}{*}{ 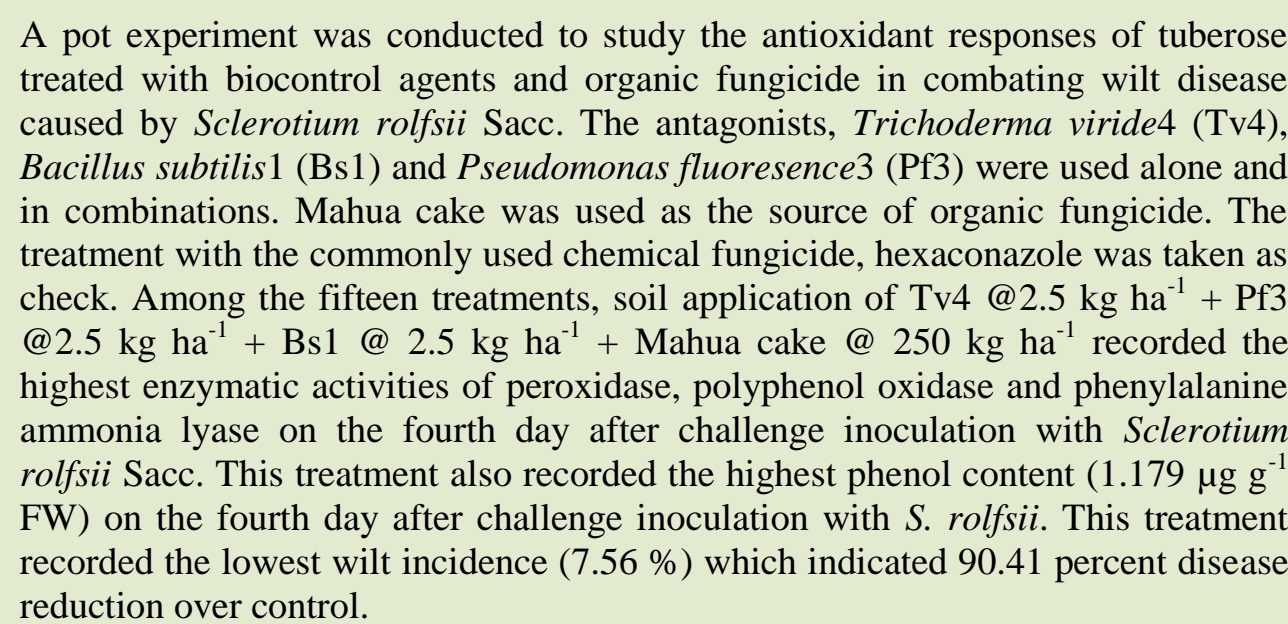 } \\
\hline & \\
\hline & \\
\hline $\begin{array}{l}\text { Polyphenol oxidase, } \\
\text { Sclerotium rolfsii, } \\
\text { Tuberose. }\end{array}$ & \\
\hline Article Info & \\
\hline $\begin{array}{l}\text { Accepted: } \\
\text { 04 May } 2017\end{array}$ & \\
\hline $\begin{array}{l}\text { Available Online: } \\
\text { 10 June } 2017\end{array}$ & \\
\hline & \\
\hline
\end{tabular}

\section{Introduction}

Tuberose (Polianthes tuberosa L.) is a commercially important ornamental bulbulous plant cultivated in India for cut and loose flower trade and also for the extraction of its highly valued natural flower oil which is one of the most expensive raw materials of highgrade perfumes. It is native of Mexico, from where it has spread to different parts of world and is now one of the most important ornamentals of tropical and sub-tropical areas. It is used for artistic garland, floral ornaments, bouquets and buttonholes. The long flower spikes are excellent as cut flowers for table decoration (Padagunur et al., 2005).

Tuberose is often attacked by Sclerotium rolfsii Sacc. Which is a ubiquitous endemic soil borne plant pathogen. The initial symptom of the disease is flaccidity and drooping of leaves. The leaves become yellow and dry up. The fungus mainly affects the roots and the infection gradually spreads upward through the tuber and collar portion of the stem. Both tubers and roots show rotting 
symptoms. Thick cottony growth of the fungus is visible on the rotten stem and on petioles at the soil level. Hexaconazole is a systemic fungicide used for the control of Sclerotium rolfsii (Virupakshaprabhu and Hiremath, 2003). But, chemical control is not always effective and there is concern about the side effects of these fungicides on the environmental safety. Natural plant products and biological control, using antagonistic microorganisms, is an alternate approach to control the pathogenic attack. Biological control of soil borne pathogens offers environmentally safe alternative to chemicals. Different species of bacteria Bacillus subtilis, Pseudomonas fluoresence and fungi Trichoderma viride are reported to be effective biocontrol agents against soil borne plant pathogens (Sivasakthi et al., 2014; Zape et al., 2014).

Plants are known to produce various stress compounds when they are exposed to the pathogens (Lavania et al., 2006; Kim et al., 2008). Studies have shown that plants possess an effective antioxidant machinery to combat disease causing pathogen attack (Demidchik, 2012). Activation of a wide array of defense responses slows down or halts infection at certain stages of the host-pathogen interaction. An increase in the activities of phenolics related enzymes and the accumulation of phenolics has been correlated to plant resistance to biotic stresses (Anjum et al., 2012). This study was conducted to explore the changes in some enzyme activities and phenol content in tuberose plants treated with bio-control and organic fungicide after inoculation with Sclerotium rolfsii.

\section{Materials and Methods}

\section{Pathogen source}

Six pathogenic isolates of Sclerotium rolfsii Sacc. were isolated from the diseased tuberose plants collected from the six different tuberose growing areas of Madurai, Dindigul, Dharmapuri and Sivagangai districts of Tamil Nadu. These isolates produced the typical wilt symptoms on the artificially inoculated tuberose plants in pot culture. The degree of virulence varied among the isolates of $S$. rolfsii to cause wilt of tuberose. The most virulent isolate was selected and mass multiplied in sand maize medium and used for this study.

\section{Plant materials and treatment}

The present investigation was carried out during November 2014 at Agricultural College and Research Institute, Madurai. Three months old tuberose plants were used for this experiment. Fifteen treatments were set up with soil application of antagonists (Tv4, Bs1, Pf3), Mahua cake and hexaconazole (Table 1). Inoculation with $S$. rolfsii was done and the leaves were collected from the plants on the $0,2,4,6$ and 10 days after inoculation. Leaves were washed several times with sterile distilled water and used for enzyme assays and phenol estimation.

\section{Enzyme assay}

Peroxidase activity was estimated by the method of Hammerschmidt et al., (1982). One gram of fresh leaf tissue was homogenisedwith5 $\mathrm{ml}$ of $0.1 \mathrm{M}$ phosphate buffer $\mathrm{pH} 7.0$ in a pre-cooled pestle and mortar. The homogenate was centrifuged at $15,000 \mathrm{rpm}$ at $4^{\circ} \mathrm{C}$ for 15 minutes.

The supernatant was used as enzyme source. The reaction mixture consisted of $1.5 \mathrm{ml}$ of $0.05 \mathrm{M}$ pyrogallol, $0.1 \mathrm{ml}$ of enzyme extract and $0.5 \mathrm{ml}$ of $1 \% \mathrm{H}_{2} \mathrm{O}_{2}$. The change in absorbance of the reaction mixture was recorded at $420 \mathrm{~nm}$ at 30 seconds interval for three minutes at room temperature. The boiled enzyme preparation served as blank. The 
enzyme activity was expressed as change in absorbance $\min ^{-1} \mathrm{~g}^{-1} \mathrm{FW}$.

Polyphenol oxidase was assayed by the method adopted by Mayer et al., (1965). One $\mathrm{g}$ of fresh leaf sample was homogenised in 1 $\mathrm{ml}$ of $0.1 \mathrm{M}$ sodium phosphate buffer $(\mathrm{pH}$ 6.5). The homogenate was centrifuged at $15,000 \mathrm{rpm}$ for 15 minutes at $4^{\circ} \mathrm{C}$ and the supernatant was used as the enzyme source. The reaction mixture consisted of $1.5 \mathrm{ml}$ of $0.1 \mathrm{M}$ sodium phosphate buffer $\mathrm{pH} 6.5$ and 0.1 $\mathrm{ml}$ of the enzyme extract. The reaction was initiated by the addition of $0.2 \mathrm{ml}$ of catechol $(0.01 \mathrm{M})$. The absorbance at $495 \mathrm{~nm}$ at $30 \mathrm{sec}$ intervals for three minutes was recorded. The enzyme activity was expressed as change in absorbance $\min ^{-1} \mathrm{~g}^{-1} \mathrm{FW}$.

The activity of phenylalanine ammonia lyase was estimated by the method of Dickerson et al., (1984). Five hundred $\mathrm{mg}$ of leaf was homogenized in $5 \mathrm{ml}$ of cold $25 \mathrm{mM}$ borate $\mathrm{HCl}$ buffer ( $\mathrm{pH}$ 8.8) containing $5 \mathrm{mM}$ mercaptoethanol. The homogenate was centrifuged at $15,000 \mathrm{rpm}$ for 15 minutes at $4^{\circ} \mathrm{C}$ and the supernatant was used as enzyme source. The assay mixture consisted of $0.2 \mathrm{ml}$ of enzyme extract, $1.3 \mathrm{ml}$ water and $0.5 \mathrm{ml}$ borate buffer. The reaction was initiated by the addition of $1 \mathrm{ml}$ of $12 \mathrm{mM} \mathrm{L}$ Phenylalanine. The reaction mixture was incubated for one hour at $32^{\circ} \mathrm{C}$. The reaction was stopped by the addition of $0.5 \mathrm{ml}$ of $2 \mathrm{~N}$ $\mathrm{HCl}$. A blank was run in which phenylalanine was added after adding $2 \mathrm{~N} \mathrm{HCl}$. The absorbance was measured at $290 \mathrm{~nm}$. The enzyme activity was expressed as $\square$ mol trans cinnamic acid $\min ^{-1} \mathrm{~g}^{-1} \mathrm{FW}$.

\section{Estimation of total phenols}

One $g$ of the leaf sample was ground well in a pestle and mortar after adding $10 \mathrm{ml}$ of $80 \%$ methanol. The homogenate was centrifuged at $10,000 \mathrm{rpm}$ for $20 \mathrm{~min}$. The supernatant was collected and evaporated to dryness and the residue was dissolved in $5 \mathrm{ml}$ of distilled water. From this, $0.2 \mathrm{ml}$ was taken and the volume was made up to $3 \mathrm{ml}$ with distilled water and to that $0.25 \mathrm{ml}$ of Folin-Ciocalteau reagent $(1 \mathrm{~N})$ was added. After three minutes, one $\mathrm{ml}$ of $20 \%$ sodium carbonate was added and placed in boiling water bath for one min and cooled. The absorbance was measured at $725 \mathrm{~nm}$ against a reagent blank. The total phenol content was expressed as $\mu \mathrm{g}$ catechol $\mathrm{g}^{-1} \mathrm{FW}$ (Zieslin and Ben Zaken, 1993).

\section{Results and Discussion}

\section{Enzyme activities}

Peroxidases (PO) has been implicated in a number of diverse phenomena observed in plants such as lignification, suberization, cell elongation, growth and regulation of cell wall biosynthesis and plasticity, which diversified during disease period (Chanda and Singh, 1997). This enzyme is also known to produce a toxic environment for the pathogens through the production of oxidative burst (Passardi et al., 2005).

The activity of peroxidase was induced in the plants treated with biocontrol agents, organic amendments and challenge inoculation with the pathogen $S$. rolfsii. The results revealed that the activity of PO was significantly higher in tuberose plants treated with consortial formulation of Tv4@2.5 kg ha ${ }^{-1}+$ Pf3@2.5 kg ha ${ }^{-1}+$ Bs1@ $2.5 \mathrm{~kg} \mathrm{ha}^{-1}+$ Mahua cake@250 kg ha ${ }^{-1}$ (0.849 change in absorbance $\min ^{-1} \mathrm{~g}^{-1} \mathrm{FW}$ ) at four days after challenge inoculation with $S$. rolfsii (Table 1). Similar result was reported by Karthikeyan et al., (2008).

The accumulation of polyphenol oxidase (PPO) plays an important role in plants defense mechanism for inhibition of pathogens by the mechanism of cell wall reinforcements (Ngadze et al., 2012). PPO is a nuclear encoded enzyme that catalyzes the 
oxygen dependent oxidation of phenols to quinones. PPOs are considered to have broad antimicrobial properties. In the present study, an increasing trend of PPO activity was seen up to four days after inoculation with $S$. rolfsii in all treatments and then decreased (Table 2).
The treatment Tv4@2.5 kg ha ${ }^{-1}+$ Pf3@2.5 $\mathrm{kg} \mathrm{ha}^{-1}+$ Bs1@2.5 kg ha ${ }^{-1}+$ Mahua cake @ $250 \mathrm{~kg} \mathrm{ha}^{-1}$ recorded the maximum (1.289 change in absorbance $\min ^{-1} \mathrm{~g}^{-1} \mathrm{FW}$ ) PPO activity as compared to the other treatments in tuberose plants.

Table.1 Changes in peroxidase (PO) activity in tuberose plants inoculated with $S$. rolfsii and treated with soil application of antagonists and organic fungicide

\begin{tabular}{|c|c|c|c|c|c|c|c|}
\hline \multirow{3}{*}{ T. No. } & \multirow{3}{*}{\multicolumn{2}{|c|}{ Treatment details }} & \multirow{2}{*}{\multicolumn{5}{|c|}{$\begin{array}{l}\text { PO activity } \\
\left(\text { Change in absorbance } \mathrm{min}^{-1} \mathrm{~g}^{-1} \mathrm{FW} \text { )* }\right. \\
\text { Davs after inoculation }\end{array}$}} \\
\hline & & & & & & & \\
\hline & & & 0 & 2 & 4 & 6 & 10 \\
\hline \multirow{2}{*}{$\mathrm{T}_{1}$} & \multirow{2}{*}{$\operatorname{Tv}_{4}\left(2.5 \mathrm{~kg} \mathrm{ha}^{-1}\right)$} & Inoculated & 0.372 & 0.589 & 0.693 & 0.659 & 0.485 \\
\hline & & Healthy & 0.201 & 0.331 & 0.289 & 0.221 & 0.223 \\
\hline \multirow{2}{*}{$\mathrm{T}_{2}$} & \multirow{2}{*}{$\mathrm{Pf}_{3}\left(2.5 \mathrm{~kg} \mathrm{ha}^{-1}\right)$} & Inoculated & 0.361 & 0.487 & 0.678 & 0.653 & 0.466 \\
\hline & & Healthy & 0.187 & 0.329 & 0.388 & 0.344 & 0.234 \\
\hline \multirow{2}{*}{$\mathrm{T}_{3}$} & \multirow{2}{*}{$\mathrm{Bs}_{1}\left(2.5 \mathrm{~kg} \mathrm{ha}^{-1}\right)$} & Inoculated & 0.296 & 0.478 & 0.589 & 0.538 & 0.418 \\
\hline & & Healthy & 0.129 & 0.278 & 0.372 & 0.279 & 0.179 \\
\hline \multirow{2}{*}{$\mathrm{T}_{4}$} & \multirow{2}{*}{ Mahua cake (250 kg ha' $\left.{ }^{-1}\right)$} & Inoculated & 0.384 & 0.546 & 0.741 & 0.696 & 0.482 \\
\hline & & Healthy & 0.198 & 0.352 & 0.456 & 0.424 & 0.346 \\
\hline \multirow{2}{*}{$\mathrm{T}_{5}$} & \multirow{2}{*}{$\mathrm{Tv}_{4}\left(2.5 \mathrm{~kg} \mathrm{ha}^{-1}\right)+\mathrm{Pf}_{3}\left(2.5 \mathrm{~kg} \mathrm{ha}^{-1}\right)$} & Inoculated & 0.351 & 0.523 & 0.710 & 0.672 & 0.559 \\
\hline & & Healthy & 0.232 & 0.364 & 0.453 & 0.371 & 0.262 \\
\hline \multirow{2}{*}{$\mathrm{T}_{6}$} & \multirow{2}{*}{$\mathrm{Pf}_{3}\left(2.5 \mathrm{~kg} \mathrm{ha}^{-1}\right)+\mathrm{Bs}_{1}\left(2.5 \mathrm{~kg} \mathrm{ha}^{-1}\right)$} & Inoculated & 0.378 & 0.586 & 0.724 & 0.702 & 0.495 \\
\hline & & Healthy & 0.182 & 0.229 & 0.347 & 0.253 & 0.197 \\
\hline \multirow{2}{*}{$\mathrm{T}_{7}$} & \multirow{2}{*}{$\mathrm{Tv}_{4}\left(2.5 \mathrm{~kg} \mathrm{ha}^{-1}\right)+\mathrm{Bs}_{1}\left(2.5 \mathrm{~kg} \mathrm{ha}^{-1}\right)$} & Inoculated & 0.389 & 0.568 & 0.733 & 0.706 & 0.563 \\
\hline & & Healthy & 0.238 & 0.252 & 0.327 & 0.289 & 0.154 \\
\hline \multirow{2}{*}{$\mathrm{T}_{8}$} & \multirow{2}{*}{$\begin{array}{l}\operatorname{Tv}_{4}\left(2.5 \mathrm{~kg} \mathrm{ha}^{-1}\right)+\mathrm{Pf}_{3}\left(2.5 \mathrm{~kg} \mathrm{ha}^{-1}\right)+\mathrm{Bs}_{1}(2.5 \\
\left.\mathrm{kg} \mathrm{ha}^{-1}\right)\end{array}$} & Inoculated & 0.331 & 0.521 & 0.702 & 0.684 & 0.423 \\
\hline & & Healthy & 0.288 & 0.381 & 0.456 & 0.327 & 0.232 \\
\hline \multirow{2}{*}{$\mathrm{T}_{9}$} & \multirow{2}{*}{$\begin{array}{l}\operatorname{Tv}_{4}\left(2.5 \mathrm{~kg} \mathrm{ha}^{-1}\right)+\mathrm{Pf}_{3}\left(2.5 \mathrm{~kg} \mathrm{ha}^{-1}\right)+\text { Mahua } \\
\text { cake }\left(250 \mathrm{~kg} \mathrm{ha}^{-1}\right)\end{array}$} & Inoculated & 0.322 & 0.467 & 0.798 & 0.665 & 0.479 \\
\hline & & \begin{tabular}{|l|} 
Healthy \\
\end{tabular} & 0.203 & 0.284 & 0.347 & 0.264 & 0.197 \\
\hline \multirow{2}{*}{$\mathrm{T}_{10}$} & \multirow{2}{*}{$\begin{array}{l}\mathrm{Pf}_{3}\left(2.5 \mathrm{~kg} \mathrm{ha}^{-1}\right)+\mathrm{Bs}_{1}\left(2.5 \mathrm{~kg} \mathrm{ha}^{-1}\right)+\text { Mahua } \\
\text { cake }\left(250 \mathrm{~kg} \mathrm{ha}^{-1}\right)\end{array}$} & Inoculated & 0.357 & 0.427 & 0.678 & 0.636 & 0.346 \\
\hline & & Healthy & 0.212 & 0.288 & 0.393 & 0.343 & 0.212 \\
\hline \multirow{2}{*}{$\mathrm{T}_{11}$} & \multirow{2}{*}{$\begin{array}{l}\operatorname{Tv}_{4}\left(2.5 \mathrm{~kg} \mathrm{ha}^{-1}\right)+\mathrm{Bs}_{1}\left(2.5 \mathrm{~kg} \mathrm{ha}^{-1}\right)+\text { Mahua } \\
\text { cake }\left(250 \mathrm{~kg} \mathrm{ha}^{-1}\right)\end{array}$} & Inoculated & 0.322 & 0.418 & 0.587 & 0.539 & 0.398 \\
\hline & & Healthy & 0.232 & 0.257 & 0.293 & 0.221 & 0.122 \\
\hline \multirow{2}{*}{$\mathrm{T}_{12}$} & \multirow{2}{*}{$\begin{array}{l}\operatorname{Tv}_{4}\left(2.5 \mathrm{~kg} \mathrm{ha}^{-1}\right)+\mathrm{Pf}_{3}\left(2.5 \mathrm{~kg} \mathrm{ha}^{-1}\right)+\mathrm{Bs}_{1}(2.5 \\
\left.\mathrm{kg} \mathrm{ha}^{-1}\right)+ \text { Mahua cake }\left(250 \mathrm{~kg} \mathrm{ha}^{-1}\right)\end{array}$} & Inoculated & 0.418 & 0.647 & \begin{tabular}{|l|}
0.849 \\
\end{tabular} & 0.789 & 0.523 \\
\hline & & Healthy & 0.245 & 0.319 & 0.452 & 0.363 & 0.245 \\
\hline \multirow{2}{*}{$\mathrm{T}_{13}$} & & Inoculated & 0.373 & 0.591 & 0.826 & 0.796 & 0.528 \\
\hline & Hexaconazole $(0.1 \%)$ & Healthy & 0.219 & 0.322 & 0.668 & 0.443 & 0.294 \\
\hline $\mathrm{T}_{14}$ & Control (Inoculated) & & 0.329 & 0.366 & 0.501 & 0.479 & 0.276 \\
\hline $\mathrm{T}_{15}$ & Healthy control & & 0.336 & 0.387 & 0.519 & 0.491 & 0.288 \\
\hline & $\begin{array}{l}\mathrm{CD}(\mathrm{p}=0.05) \\
\text { Treatments } 0.007 \\
\text { Days } 0.004 \\
\text { Treatment x Days } 0.017\end{array}$ & & & & & & \\
\hline
\end{tabular}

\footnotetext{
*All values are means of three replications
} 
Table.2 Changes in polyphenol oxidase (PPO) activity in tuberose plants inoculated with S.rolfsii and treated with soil application of antagonists and organic fungicide

\begin{tabular}{|c|c|c|c|c|c|c|c|}
\hline \multirow{3}{*}{ T.No. } & \multirow{3}{*}{\multicolumn{2}{|c|}{ Treatment details }} & \multirow{2}{*}{\multicolumn{5}{|c|}{$\begin{array}{l}\text { PPO activity } \\
\left.\text { (Change in absorbance } \min ^{-1} \mathrm{~g}^{-1} \mathrm{FW}\right)^{*} \\
\text { Davs after inoculation }\end{array}$}} \\
\hline & & & & & & & \\
\hline & & & 0 & 2 & 4 & 6 & 10 \\
\hline \multirow{2}{*}{$\mathrm{T}_{1}$} & \multirow{2}{*}{$\operatorname{Tv}_{4}\left(2.5 \mathrm{~kg} \mathrm{ha}^{-1}\right)$} & Inoculated & 0.443 & 0.589 & 0.788 & 0.643 & 0.603 \\
\hline & & Healthy & 0.418 & 0.478 & 0.701 & 0.598 & 0.475 \\
\hline \multirow{2}{*}{$\mathrm{T}_{2}$} & \multirow{2}{*}{$\mathrm{Pf}_{3}\left(2.5 \mathrm{~kg} \mathrm{ha}^{-1}\right)$} & Inoculated & 0.438 & 0.583 & 0.788 & 0.649 & 0.607 \\
\hline & & Healthy & 0.366 & 0.487 & 0.701 & 0.608 & 0.575 \\
\hline \multirow{2}{*}{$\mathrm{T}_{3}$} & \multirow{2}{*}{$\mathrm{Bs}_{1}\left(2.5 \mathrm{~kg} \mathrm{ha}^{-1}\right)$} & Inoculated & 0.419 & 0.432 & 0.674 & 0.610 & 0.573 \\
\hline & & Healthy & 0.389 & 0.407 & 0.546 & 0.512 & 0.464 \\
\hline \multirow{2}{*}{$\mathrm{T}_{4}$} & \multirow{2}{*}{ Mahua cake $\left(250 \mathrm{~kg} \mathrm{ha}^{-1}\right)$} & Inoculated & 0.464 & 0.632 & 0.806 & 0.735 & 0.536 \\
\hline & & Healthy & 0.412 & 0.578 & 0.739 & 0.662 & 0.484 \\
\hline \multirow{2}{*}{$\mathrm{T}_{5}$} & \multirow{2}{*}{$\mathrm{Tv}_{4}\left(2.5 \mathrm{~kg} \mathrm{ha}^{-1}\right)+\mathrm{Pf}_{3}\left(2.5 \mathrm{~kg} \mathrm{ha}^{-1}\right)$} & Inoculated & 0.467 & 0.532 & 0.798 & 0.694 & 0.634 \\
\hline & & Healthy & 0.398 & 0.486 & 0.679 & 0.601 & 0.559 \\
\hline \multirow{2}{*}{$\mathrm{T}_{6}$} & \multirow{2}{*}{$\mathrm{Pf}_{3}\left(2.5 \mathrm{~kg} \mathrm{ha}^{-1}\right)+\mathrm{Bs}_{1}\left(2.5 \mathrm{~kg} \mathrm{ha}^{-1}\right)$} & Inoculated & 0.474 & 0.989 & 1.018 & 0.752 & 0.549 \\
\hline & & Healthy & 0.423 & 0.910 & 0.932 & 0.634 & 0.488 \\
\hline \multirow{2}{*}{$\mathrm{T}_{7}$} & \multirow{2}{*}{$\mathrm{Tv}_{4}\left(2.5 \mathrm{~kg} \mathrm{ha}^{-1}\right)+\mathrm{Bs}_{1}\left(2.5 \mathrm{~kg} \mathrm{ha}^{-1}\right)$} & Inoculated & 0.446 & 0.785 & 0.943 & 0.715 & 0.517 \\
\hline & & Healthy & 0.412 & 0.702 & 0.821 & 0.669 & 0.436 \\
\hline \multirow{2}{*}{$\mathrm{T}_{8}$} & \multirow{2}{*}{$\begin{array}{l}\mathrm{Tv}_{4}\left(2.5 \mathrm{~kg} \mathrm{ha}^{-1}\right)+\mathrm{Pf}_{3}\left(2.5 \mathrm{~kg} \mathrm{ha}^{-1}\right)+\mathrm{Bs}_{1} \\
\left(2.5 \mathrm{~kg} \mathrm{ha}^{-1}\right)\end{array}$} & Inoculated & 0.423 & 0.656 & 0.832 & 0.698 & 0.626 \\
\hline & & Healthy & 0.388 & 0.553 & 0.797 & 0.558 & 0.516 \\
\hline \multirow{2}{*}{$\mathrm{T}_{9}$} & \multirow{2}{*}{$\begin{array}{l}\operatorname{Tv}_{4}\left(2.5 \mathrm{~kg} \mathrm{ha}^{-1}\right)+\mathrm{Pf}_{3}\left(2.5 \mathrm{~kg} \mathrm{ha}^{-1}\right)+\text { Mahua } \\
\text { cake }\left(250 \mathrm{~kg} \mathrm{ha}^{-1}\right)\end{array}$} & Inoculated & 0.476 & 0.772 & 0.859 & 0.683 & 0.615 \\
\hline & & Healthy & 0.422 & 0.537 & 0.798 & 0.542 & 0.521 \\
\hline \multirow{2}{*}{$\mathrm{T}_{10}$} & \multirow{2}{*}{$\begin{array}{l}\mathrm{Pf}_{3}\left(2.5 \mathrm{~kg} \mathrm{ha}^{-1}\right)+\mathrm{Bs}_{1}\left(2.5 \mathrm{~kg} \mathrm{ha}^{-1}\right)+\text { Mahua } \\
\text { cake }\left(250 \mathrm{~kg} \mathrm{ha}^{-1}\right)\end{array}$} & Inoculated & 0.467 & 0.679 & 0.812 & 0.648 & 0.632 \\
\hline & & Healthy & 0.416 & 0.523 & 0.735 & 0.463 & 0.412 \\
\hline \multirow{2}{*}{$\mathrm{T}_{11}$} & \multirow{2}{*}{$\begin{array}{l}\operatorname{Tv}_{4}\left(2.5 \mathrm{~kg} \mathrm{ha}^{-1}\right)+\mathrm{Bs}_{1}\left(2.5 \mathrm{~kg} \mathrm{ha}^{-1}\right)+ \\
\text { Mahua cake }\left(250 \mathrm{~kg} \mathrm{ha}^{-1}\right)\end{array}$} & Inoculated & 0.425 & 0.498 & 0.791 & 0.647 & 0.472 \\
\hline & & Healthy & 0.386 & 0.399 & 0.637 & 0.598 & 0.313 \\
\hline \multirow{2}{*}{$\mathrm{T}_{12}$} & \multirow{2}{*}{$\begin{array}{l}\operatorname{Tv}_{4}\left(2.5 \mathrm{~kg} \mathrm{ha}^{-1}\right)+\mathrm{Pf}_{3}\left(2.5 \mathrm{~kg} \mathrm{ha}^{-1}\right)+\mathrm{Bs}_{1} \\
\left(2.5 \mathrm{~kg} \mathrm{ha}^{-1}\right)+\text { Mahua cake }\left(250 \mathrm{~kg} \mathrm{ha}^{-1}\right)\end{array}$} & Inoculated & 0.994 & 1.177 & 1.289 & 0.920 & 0.618 \\
\hline & & Healthy & 0.883 & 0.948 & 1.022 & 0.757 & 0.485 \\
\hline \multirow{2}{*}{$\mathrm{T}_{13}$} & Ho & Inoculated & 0.493 & 0.735 & 0.957 & 0.884 & 0.816 \\
\hline & Hexaconazoie(0.1\%) & Healthy & 0.423 & 0.676 & 0.848 & 0.788 & 0.456 \\
\hline $\mathrm{T}_{14}$ & Control (Inoculated) & & 0.402 & 0.425 & 0.605 & 0.479 & 0.308 \\
\hline $\mathrm{T}_{15}$ & Healthy control & & 0.414 & 0.423 & 0.658 & 0.506 & 0.347 \\
\hline & $\begin{array}{l}\mathrm{CD}(\mathrm{p}=0.05) \\
\text { Treatments } 0.011 \\
\text { Days } 0.006 \\
\text { Treatment x Days } 0.026\end{array}$ & & & & & & \\
\hline
\end{tabular}

*All values are means of three replications 
Table.3 Changes in phenylalanine ammonia lyase (PAL) activity in tuberose plants inoculated with $S$. rolfsii and treated with soil application of antagonists and organic fungicide

\begin{tabular}{|c|c|c|c|c|c|c|c|}
\hline \multirow{3}{*}{ T.No } & \multirow{3}{*}{\multicolumn{2}{|c|}{ Treatment details }} & \multirow{2}{*}{\multicolumn{5}{|c|}{$\begin{array}{l}\text { PAL activity } \\
\left(\mu \mathrm{mol} \text { trans-cinnamic acid } \mathrm{min}^{-1} \mathrm{~g}^{-1} \mathrm{FW}\right)^{*} \\
\text { Davs after inoculation }\end{array}$}} \\
\hline & & & & & & & \\
\hline & & & 0 & 2 & 4 & 6 & $\mathbf{1 0}$ \\
\hline \multirow{2}{*}{$\mathrm{T}_{1}$} & \multirow{2}{*}{$\operatorname{Tv}_{4}\left(2.5 \mathrm{~kg} \mathrm{ha}^{-1}\right)$} & Inoculated & 0.462 & 0.588 & 0.697 & 0.686 & 0.604 \\
\hline & & Healthy & 0.323 & 0.546 & 0.589 & 0.538 & 0.424 \\
\hline \multirow{2}{*}{$\mathrm{T}_{2}$} & \multirow{2}{*}{$\mathrm{Pf}_{3}\left(2.5 \mathrm{~kg} \mathrm{ha}^{-1}\right)$} & Inoculated & 0.412 & 0.517 & 0.663 & 0.645 & 0.593 \\
\hline & & Healthy & 0.378 & 0.486 & 0.545 & 0.328 & 0.234 \\
\hline \multirow{2}{*}{$\mathrm{T}_{3}$} & \multirow{2}{*}{$\mathrm{Bs}_{1}\left(2.5 \mathrm{~kg} \mathrm{ha}^{-1}\right)$} & Inoculated & 0.436 & 0.486 & 0.583 & 0.541 & 0.487 \\
\hline & & Healthy & 0.369 & 0.384 & 0.589 & 0.532 & 0.424 \\
\hline \multirow{2}{*}{$\mathrm{T}_{4}$} & \multirow{2}{*}{ Mahua cake $\left(250 \mathrm{~kg} \mathrm{ha}^{-1}\right)$} & Inoculated & 0.478 & 0.523 & 0.721 & 0.702 & 0.676 \\
\hline & & Healthy & 0.388 & 0.472 & 0.681 & 0.568 & 0.538 \\
\hline \multirow{2}{*}{$\mathrm{T}_{5}$} & \multirow{2}{*}{$\operatorname{Tv}_{4}\left(2.5 \mathrm{~kg} \mathrm{ha}^{-1}\right)+\mathrm{Pf}_{3}\left(2.5 \mathrm{~kg} \mathrm{ha}^{-1}\right)$} & Inoculated & 0.463 & 0.498 & 0.707 & 0.626 & 0.578 \\
\hline & & Healthy & 0.425 & 0.436 & 0.688 & 0.596 & 0.482 \\
\hline \multirow{2}{*}{$\mathrm{T}_{6}$} & \multirow{2}{*}{$\mathrm{Pf}_{3}\left(2.5 \mathrm{~kg} \mathrm{ha}^{-1}\right)+\mathrm{Bs}_{1}\left(2.5 \mathrm{~kg} \mathrm{ha}^{-1}\right)$} & Inoculated & 0.481 & 0.578 & 0.745 & 0.686 & 0.648 \\
\hline & & Healthy & 0.416 & 0.526 & 0.692 & 0.578 & 0.498 \\
\hline \multirow{2}{*}{$\mathrm{T}_{7}$} & \multirow{2}{*}{$\mathrm{Tv}_{4}\left(2.5 \mathrm{~kg} \mathrm{ha}^{-1}\right)+\mathrm{Bs}_{1}\left(2.5 \mathrm{~kg} \mathrm{ha}^{-1}\right)$} & Inoculated & 0.462 & 0.524 & 0.737 & 0.678 & 0.536 \\
\hline & & Healthy & 0.428 & 0.469 & 0.688 & 0.567 & 0.436 \\
\hline \multirow{2}{*}{$\mathrm{T}_{8}$} & \multirow{2}{*}{$\begin{array}{l}\operatorname{Tv}_{4}\left(2.5 \mathrm{~kg} \mathrm{ha}^{-1}\right)+\mathrm{Pf}_{3}\left(2.5 \mathrm{~kg} \mathrm{ha}^{-1}\right)+\mathrm{Bs}_{1} \\
\left(2.5 \mathrm{~kg} \mathrm{ha}^{-1}\right)\end{array}$} & Inoculated & 0.434 & 0.512 & 0.726 & 0.632 & 0.512 \\
\hline & & Healthy & 0.386 & 0.464 & 0.679 & 0.588 & 0.448 \\
\hline \multirow{2}{*}{$\mathrm{T}_{9}$} & \multirow{2}{*}{$\begin{array}{l}\operatorname{Tv}_{4}\left(2.5 \mathrm{~kg} \mathrm{ha}^{-1}\right)+\mathrm{Pf}_{3}\left(2.5 \mathrm{~kg} \mathrm{ha}^{-1}\right)+ \\
\text { Mahua cake }\left(250 \mathrm{~kg} \mathrm{ha}^{-1}\right)\end{array}$} & Inoculated & 0.416 & 0.507 & 0.714 & 0.608 & 0.538 \\
\hline & & Healthy & 0.389 & 0.462 & 0.684 & 0.546 & 0.462 \\
\hline \multirow{2}{*}{$\mathrm{T}_{10}$} & \multirow{2}{*}{$\begin{array}{l}\mathrm{Pf}_{3}\left(2.5 \mathrm{~kg} \mathrm{ha}^{-1}\right)+\mathrm{Bs}_{1}\left(2.5 \mathrm{~kg} \mathrm{ha}^{-1}\right)+ \\
\text { Mahua cake }\left(250 \mathrm{~kg} \mathrm{ha}^{-1}\right)\end{array}$} & Inoculated & 0.407 & 0.473 & 0.711 & 0.567 & 0.523 \\
\hline & & Healthy & 0.326 & 0.387 & 0.674 & 0.558 & 0.477 \\
\hline \multirow{2}{*}{$\mathrm{T}_{11}$} & \multirow{2}{*}{$\begin{array}{l}\mathrm{Tv}_{4}\left(2.5 \mathrm{~kg} \mathrm{ha}^{-1}\right)+\mathrm{Bs}_{1}\left(2.5 \mathrm{~kg} \mathrm{ha}^{-1}\right)+ \\
\text { Mahua cake }\left(250 \mathrm{~kg} \mathrm{ha}^{-1}\right)\end{array}$} & Inoculated & 0.392 & 0.442 & 0.708 & 0.519 & 0.473 \\
\hline & & Healthy & 0.323 & 0.344 & 0.689 & 0.483 & 0.425 \\
\hline \multirow{2}{*}{$\mathrm{T}_{12}$} & \multirow{2}{*}{$\begin{array}{l}\operatorname{Tv}_{4}\left(2.5 \mathrm{~kg} \mathrm{ha}^{-1}\right)+\mathrm{Pf}_{3}\left(2.5 \mathrm{~kg} \mathrm{ha}^{-1}\right)+\mathrm{Bs}_{1} \\
\left(2.5 \mathrm{~kg} \mathrm{ha}^{-1}\right)+\text { Mahua cake }\left(250 \mathrm{~kg} \mathrm{ha}^{-1}\right)\end{array}$} & Inoculated & 0.507 & 0.686 & 0.872 & 0.732 & 0.687 \\
\hline & & Healthy & 0.428 & 0.567 & 0.736 & 0.687 & 0.632 \\
\hline \multirow{2}{*}{$\mathrm{T}_{13}$} & Ho 50 o 100 & Inoculated & 0.491 & 0.533 & 0.732 & 0.712 & 0.646 \\
\hline & Hexaconazore (0.1\%) & Healthy & 0.367 & 0.437 & 0.658 & 0.646 & 0.548 \\
\hline $\mathrm{T}_{14}$ & Control (Inoculated) & & 0.398 & 0.516 & 0.704 & 0.579 & 0.426 \\
\hline $\mathrm{T}_{15}$ & Healthy control & & 0.384 & 0.426 & 0.628 & 0.433 & 0.403 \\
\hline & $\begin{array}{l}\mathrm{CD}(\mathrm{p}=0.05) \\
\text { Treatments } 0.010 \\
\text { Days } 0.005 \\
\text { Treatment x Days } 0.022\end{array}$ & & & & & & \\
\hline
\end{tabular}

\footnotetext{
*All values are means of three replications
} 
Table.4 Changes in total phenolic content in tuberose plants inoculated with $S$. rolfsii and treated with soil application of antagonists and organic fungicide

\begin{tabular}{|c|c|c|c|c|c|c|c|}
\hline \multirow{3}{*}{ T.No. } & \multirow{3}{*}{\multicolumn{2}{|c|}{ Treatment details }} & \multirow{2}{*}{\multicolumn{5}{|c|}{ 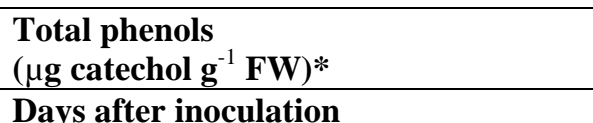 }} \\
\hline & & & & & & & \\
\hline & & & $\mathbf{0}$ & 2 & 4 & 6 & $\mathbf{1 0}$ \\
\hline \multirow{2}{*}{$\mathrm{T}_{1}$} & \multirow{2}{*}{$\operatorname{Tv}_{4}\left(2.5 \mathrm{~kg} \mathrm{ha}^{-1}\right)$} & Inoculated & 0.453 & 0.588 & 0.789 & 0.646 & 0.606 \\
\hline & & Healthy & 0.412 & 0.482 & 0.711 & 0.596 & 0.473 \\
\hline \multirow{2}{*}{$\mathrm{T}_{2}$} & \multirow{2}{*}{$\mathrm{Pf}_{3}\left(2.5 \mathrm{~kg} \mathrm{ha}^{-1}\right)$} & Inoculated & 0.435 & 0.582 & 0.786 & 0.648 & 0.602 \\
\hline & & Healthy & 0.368 & 0.486 & 0.703 & 0.608 & 0.576 \\
\hline \multirow{2}{*}{$\mathrm{T}_{3}$} & \multirow{2}{*}{$\mathrm{Bs}_{1}\left(2.5 \mathrm{~kg} \mathrm{ha}^{-1}\right)$} & Inoculated & 0.418 & 0.431 & 0.676 & 0.615 & 0.573 \\
\hline & & Healthy & 0.389 & 0.408 & 0.544 & 0.516 & 0.466 \\
\hline \multirow{2}{*}{$\mathrm{T}_{4}$} & \multirow{2}{*}{ Mahua cake $\left(250 \mathrm{~kg} \mathrm{ha}^{-1}\right)$} & Inoculated & 0.476 & 0.636 & 0.804 & 0.738 & 0.536 \\
\hline & & Healthy & 0.412 & 0.578 & 0.739 & 0.666 & 0.482 \\
\hline \multirow{2}{*}{$\mathrm{T}_{5}$} & \multirow{2}{*}{$\operatorname{Tv}_{4}\left(2.5 \mathrm{~kg} \mathrm{ha}^{-1}\right)+\mathrm{Pf}_{3}\left(2.5 \mathrm{~kg} \mathrm{ha}^{-1}\right)$} & Inoculated & 0.463 & 0.536 & 0.796 & 0.694 & 0.644 \\
\hline & & Healthy & 0.398 & 0.486 & 0.689 & 0.621 & 0.569 \\
\hline \multirow{2}{*}{$\mathrm{T}_{6}$} & \multirow{2}{*}{$\mathrm{Pf}_{3}\left(2.5 \mathrm{~kg} \mathrm{ha}^{-1}\right)+\mathrm{Bs}_{1}\left(2.5 \mathrm{~kg} \mathrm{ha}^{-1}\right)$} & Inoculated & 0.486 & 0.689 & 0.818 & 0.752 & 0.546 \\
\hline & & Healthy & 0.423 & 0.536 & 0.732 & 0.635 & 0.484 \\
\hline \multirow{2}{*}{$\mathrm{T}_{7}$} & \multirow{2}{*}{$\mathrm{Tv}_{4}\left(2.5 \mathrm{~kg} \mathrm{ha}^{-1}\right)+\mathrm{Bs}_{1}\left(2.5 \mathrm{~kg} \mathrm{ha}^{-1}\right)$} & Inoculated & 0.442 & 0.783 & 0.643 & 0.718 & 0.516 \\
\hline & & Healthy & 0.426 & 0.702 & 0.826 & 0.669 & 0.436 \\
\hline \multirow{2}{*}{$\mathrm{T}_{8}$} & \multirow{2}{*}{$\begin{array}{l}\operatorname{Tv}_{4}\left(2.5 \mathrm{~kg} \mathrm{ha}^{-1}\right)+\mathrm{Pf}_{3}\left(2.5 \mathrm{~kg} \mathrm{ha}^{-1}\right)+\mathrm{Bs}_{1}(2.5 \\
\left.\mathrm{kg} \mathrm{ha}^{-1}\right)\end{array}$} & Inoculated & 0.438 & 0.662 & 0.834 & 0.696 & 0.628 \\
\hline & & Healthy & 0.386 & 0.552 & 0.794 & 0.568 & 0.521 \\
\hline \multirow{2}{*}{$\mathrm{T}_{9}$} & \multirow{2}{*}{$\begin{array}{l}\operatorname{Tv}_{4}\left(2.5 \mathrm{~kg} \mathrm{ha}^{-1}\right)+\mathrm{Pf}_{3}\left(2.5 \mathrm{~kg} \mathrm{ha}^{-1}\right)+\text { Mahua } \\
\text { cake }\left(250 \mathrm{~kg} \mathrm{ha}^{-1}\right)\end{array}$} & Inoculated & 0.476 & 0.772 & 0.859 & 0.683 & 0.615 \\
\hline & & Healthy & 0.432 & 0.548 & 0.785 & 0.552 & 0.526 \\
\hline \multirow{2}{*}{$\mathrm{T}_{10}$} & \multirow{2}{*}{$\begin{array}{l}\mathrm{Pf}_{3}\left(2.5 \mathrm{~kg} \mathrm{ha}^{-1}\right)+\mathrm{Bs}_{1}\left(2.5 \mathrm{~kg} \mathrm{ha}^{-1}\right)+\text { Mahua } \\
\text { cake }\left(250 \mathrm{~kg} \mathrm{ha}^{-1}\right)\end{array}$} & Inoculated & 0.468 & 0.683 & 0.822 & 0.658 & 0.622 \\
\hline & & Healthy & 0.414 & 0.538 & 0.757 & 0.473 & 0.432 \\
\hline \multirow{2}{*}{$\mathrm{T}_{11}$} & \multirow{2}{*}{$\begin{array}{l}\operatorname{Tv}_{4}\left(2.5 \mathrm{~kg} \mathrm{ha}^{-1}\right)+\mathrm{Bs}_{1}\left(2.5 \mathrm{~kg} \mathrm{ha}^{-1}\right)+\text { Mahua } \\
\text { cake }\left(250 \mathrm{~kg} \mathrm{ha}^{-1}\right)\end{array}$} & Inoculated & 0.425 & 0.498 & 0.791 & 0.647 & 0.472 \\
\hline & & Healthy & 0.383 & 0.389 & 0.635 & 0.588 & 0.323 \\
\hline \multirow{2}{*}{$\mathrm{T}_{12}$} & \multirow{2}{*}{$\begin{array}{l}\operatorname{Tv}_{4}\left(2.5 \mathrm{~kg} \mathrm{ha}^{-1}\right)+\mathrm{Pf}_{3}\left(2.5 \mathrm{~kg} \mathrm{ha}^{-1}\right)+\mathrm{Bs}_{1}(2.5 \\
\left.\mathrm{kg} \mathrm{ha}^{-1}\right)+ \text { Mahua cake }\left(250 \mathrm{~kg} \mathrm{ha}^{-1}\right)\end{array}$} & Inoculated & 0.984 & 0.998 & 1.179 & 0.890 & 0.756 \\
\hline & & Healthy & 0.873 & 0.858 & 1.012 & 0.798 & 0.685 \\
\hline \multirow{2}{*}{$\mathrm{T}_{13}$} & Hexaconazole $(01 \%)$ & Inoculated & 0.495 & 0.734 & 0.967 & 0.882 & 0.815 \\
\hline & Hexaconazole $(0.1 \%)$ & Healthy & 0.423 & 0.676 & 0.846 & 0.786 & 0.589 \\
\hline $\mathrm{T}_{14}$ & Control (Inoculated) & & 0.403 & 0.425 & 0.606 & 0.478 & 0.310 \\
\hline $\mathrm{T}_{15}$ & Healthy control & & 0.416 & 0.434 & 0.668 & 0.536 & 0.378 \\
\hline & $\begin{array}{l}\mathrm{CD}(\mathrm{p}=0.05) \\
\text { Treatments } 0.011 \\
\text { Days } 0.006 \\
\text { Treatment x Days } 0.025\end{array}$ & & & & & & \\
\hline
\end{tabular}

*All values are means of three replications

Phenylalanine ammonia lyase (PAL) is the primary enzyme in the phenylpropanoid pathway, which leads to the conversion of Lphenylalanine to trans-cinnamic acid with the elimination of ammonia. It is the key enzyme in the synthesis of several defense related secondary compounds such as phenols and lignin (Anjum, 2012). Activity of phenylalanine ammonia lyase was induced in tuberose plants treated with effective biocontrol agents, organic fungicide and challenge inoculation with the pathogen $S$. rolfsii. The enzyme activity reached the maximum at four days after inoculation and maintained at higher level up to six days after challenge inoculation with respective pathogen and declined thereafter in all the treatments. Whereas, in healthy and pathogen inoculated control plants, the activity was less than that in the other treatments. Consortial 
formulation of biocontrol agentsTv4@2.5 kg $\mathrm{ha}^{-1}+$ Pf3@2.5 kg ha ${ }^{-1}+$ Bs1 @ $2.5 \mathrm{~kg} \mathrm{ha}^{-1}$ + Mahua cake@250 kg ha ${ }^{-1}$ recorded higher activity of PAL enzyme in plants. The maximum PAL activity $(0.872 \mu \mathrm{mol}$ of transcinamic acid $\min ^{-1} \mathrm{~g}^{-1} \mathrm{FW}$ ) was recorded at four days after challenge inoculation. Similar results were reported by Kannan and Karthik (2009) and Nandhini et al., (2010) (Table 3).

\section{Total phenols}

Phenolics are known to be involved in plantpathogen interactions. Some of the oxidized products of phenols are toxic to microorganisms. Phenols also act as antioxidants in scavenging reactive oxygen species (Demidchik, 2012).

The present study showed that total phenol content had an increasing trend up to fourth day. Consortial formulation of biocontrol agents Tv4@2.5 kg ha ${ }^{-1}+$ Pf3 @ $2.5 \mathrm{~kg} \mathrm{ha}^{-1}$ +Bs1@2.5 kg ha ${ }^{-1}+$ Mahua cake @ $250 \mathrm{~kg}$ $\mathrm{ha}^{-1}$ recorded higher phenol content in plants. The maximum amount of phenol $(1.179 \mu \mathrm{g}$ of catechol $\mathrm{g}^{-1} \mathrm{FW}$ ) was recorded at four days after challenge inoculation and then declined slowly and reached $0.756 \mu \mathrm{g}$ of catechol $\mathrm{g}^{-1}$ FW at ten days after inoculation (Table 4). Previous studies also reported similar trends (Angayarkanni, 2014).

It is concluded that defense related enzyme activity and phenol content increased when subjected to pathogen attack along with treatment with antagonists and fungicides.

The activity of defense related enzymes was higher in the treatment combination of Tv4@ $2.5 \mathrm{~kg} \mathrm{ha}^{-1}+$ Pf3@2.5 kg ha ${ }^{-1}+$ Bs1@2.5 $\mathrm{kg} \mathrm{ha}^{-1}+$ Mahua cake @250 kg ha ${ }^{-1}$ which in turn resisted the growth of pathogen $(S$. rolfsii Sacc.), obviously controlling the wilt in tuberose.

\section{References}

Anjum, T, Fatima, S. and Amjad, S. 2012. Physiological changes in wheat during development of loose smut. Tropical Plant Pathol. 37: 102-107.

Angayarkanni, T. 2014. Biomanagement of root rot and leaf spot disease of Stevia rebaudiana using plant growth promoting rhizobacteria. Shodhganga. pp 1-80.

Chanda, S.V., Singh, Y.D. 1997. Changes in peroxidase and IAA oxidase activities during wheat grain development. Plant Physiol. Biochem. 35: 245-250.

Demidchik, V. 2012. Reactive oxygen species and oxidative stress in plants In: Plant stress physiology, Shabala. S (Ed). CAB International, UK. pp 24-58.

Dickerson, D.P., Pascholati, S.F., Hagerman, A.E., Butler, L.G. and Niholson, R.L. 1984. Phenylalanine ammonia lyase and hydroxyl cinnamate: CoA ligase in maize mesocotyls inoculated with Helminthosporium maydis or Helminthosporium carbonum. Physiol. Plant Pathol. 25: 111-123.

Hammerschmidt, R., Nuckles, E.M. and Kuc, J. 1982. Association of enhanced peroxidase activity with induced systemic resistance of cucumber to Colletotrichum lagenarium. Physiol. Plant Pathol. 20: 73-82.

Kannan, C. and Karthik, M. 2009. Systemic induction of defense enzymes by rhizosphere microbes in cocoa seedlings. J. Biol. Control. 23: 427-431.

Karthikeyan, M., Radhika, K., Bhaskaran, R., Mathiyazhagan, S., Sandosskumar, R., Velazhahan, R. and Alice, D. 2008. Biological control of onion leaf blight disease by bulb and foliar application of powder formulation of antagonist mixture. Archives of Phytopathol. And Plant Protection. 41: 407 - 417. 
Kim, Y.H., Kim, C.Y., Song, W.K., Park, D.S., Kwon, S.Y., Lee, H.S., Bang, J.W. and Kwak, S.S. 2008. Over expression of sweet potato swpa4 peroxidase results in increased hydrogen peroxide production and enhances stress tolerance in tobacco. Planta. 227: 867881.

Lavania, M., Chauhan, P.S., Chauhan, S.V.S., Singh, H.B. and Nautiyal, C.S. 2006. Induction of plant defence enzymes and phenolics by treatment with plant growth-promoting rhizobacteria Serratia marcescens NBRI1213. Current Microbiol. 52: 363-368.

Mayer, A.M., Harel, E. and Shaul, R.B. 1965. Assay of catechol oxidase a critical comparison of methods. Phytochemistry. 5: 783-789.

Nandhini, D., Mohan, J.S. and Singh, G. 2010. Induction of Systemic acquired resistance in Arachis hypogea L. by Sclerotium rolfsii derived elicitors. J. Pytopathol. 158: 594-600.

Ngadze, E., Icishahayo, D., Coutinho, T.A. and van der Waals, J.E. 2012. Role of polyphenol oxidase, peroxidase, phenylalanine ammonia lyase, chlorogenic acid, and total soluble phenols in resistance of potatoes to soft rot. Plant Disease. 96: 186-192.

Passardi, F., Cosio, C., Penel, C. and Dunand, C. 2005. Peroxidases have more functions than a Swiss army knife. Plant Cell Reports. 24: 255-265.

Sivasakthi, S., Usharani, G. and Saranraj, P. 2014. Biocontrol potentiality of plant growth promoting bacteria (PGPR)Pseudomonas fluorescens and Bacillus subtilis; a review. Afr. J. Agricultural Research. 9: 1265-1277.

Virupakshaprabhu, H. and Hiremath, P.C. 2003. Biological control of collar rot of cotton caused by Sclerotium rolfsii Sacc. Karnataka J. Agriculural Sciences. 16: 576-579.

Zape, A.S., Gade, R.M., Singh, R. and Deshmukh, V.A. 2014. Efficacy of different antagonist against the Sclerotium rolfsii, Rhizoctonia solani and Fusarium solani. The Bioscan. 9: 1431-1434.

\section{How to cite this article:}

Ragavi, G., M.L. Mini, I. Yesuraja and Sethuraman, K. 2017. Induction of Enzyme Activities in Tuberose Plants Treated with Antagonists and Organic Fungicide under Artificial Inoculation of Sclerotium rolfsii Sacc.. Int.J.Curr.Microbiol.App.Sci. 6(6): 595-603. doi: https://doi.org/10.20546/ijcmas.2017.606.070 